\title{
CROSSING PERIODIC ORBITS VIA FIRST INTEGRALS
}

\author{
JAUME LLIBRE ${ }^{1}$, DURVAL JOSÉ TONON ${ }^{2}$ AND MARIANA QUEIROZ VELTER ${ }^{3}$
}

\begin{abstract}
We characterize the families of periodic orbits of two discontinuous piecewise differential systems in $\mathbb{R}^{3}$ separated by a plane using their first integrals. One of these discontinuous piecewise differential systems is formed by linear differential systems, and the other by nonlinear differential systems.
\end{abstract}

\section{IntRoduction AND STATEMENT OF THE MAIN RESUlts}

Discontinuous piecewise differential systems have been studied intensively in recent decades and this is mainly due to the fact that they provide in many applications more realistic models, as for instance in modeling electrical circuits $[3,10]$, in some mechanical problems [1], in control theory problems [2], etc. We define a discontinuous piecewise differential system following the rules of Filippov [6].

In the study of the dynamics of differential equations the periodic orbits play a main role when they exist, and the crossing periodic orbits play also a relevant role in the case of the discontinuous piecewise differential systems. A crossing periodic orbit of a discontinuous piecewise differential system in $\mathbb{R}^{3}$ is a periodic orbit which intersect the surface of discontinuity in a finite number $n$ of points with $n>1$.

The objective of this paper is to study the crossing periodic orbits of discontinuous piecewise differential systems of the form

$$
X(x, y, z)=X_{1}(x, y, z)+\operatorname{sign}(x) X_{2}(x, y, z),
$$

where $X_{1}$ and $X_{2}$ are smooth vector fields in $\mathbb{R}^{3}$ using their first integrals. As usual the sign function is defined as

$$
\operatorname{sign}(x)=\left\{\begin{aligned}
-1 & \text { if } x<0 \\
0 & \text { if } x=0 \\
1 & \text { if } x>0
\end{aligned}\right.
$$

2010 Mathematics Subject Classification. 34C27, 34J45.

Key words and phrases. Discontinuous piecewise differential systems, first integrals, periodic orbits, homoclinic orbits. 
We note that the discontinuous piecewise differential system (1) is separated by the plane $x=0$, and it is given by the system $Y=X_{1}+X_{2}$ in $x>0$, and by the system $Z=X_{1}-X_{2}$ in $x<0$. If $H_{1}$ and $H_{2}$ are first integrals of $Y$ and $Z$ respectively, then

$$
H=\frac{H_{1}+H_{2}}{2}+\operatorname{sign}(x)\left(\frac{H_{1}-H_{2}}{2}\right),
$$

is a first integral of the discontinuous piecewise differential system (1).

More precisely, we shall characterize the crossing periodic orbits having two and four points in the plane $x=0$ of the following two discontinuous piecewise differential systems. First, the discontinuous piecewise linear differential system

$$
\begin{aligned}
& \dot{x}=z, \\
& \dot{y}=z, \\
& \dot{z}=-\operatorname{sign}(x) y,
\end{aligned}
$$

which has the two independent first integrals

$$
H(x, y, z)=y^{2}+\operatorname{sign}(x) z^{2}, \quad F(x, y, z)=x-y,
$$

as it is easy to verify. Second, the discontinuous piecewise nonlinear differential system

$$
\begin{aligned}
& \dot{x}=(x-y) z, \\
& \dot{y}=z, \\
& \dot{z}=-\operatorname{sign}(x) y,
\end{aligned}
$$

which has the two independent first integrals

$$
H(x, y, z)=y^{2}+\operatorname{sign}(x) z^{2}, \quad G(x, y, z)=e^{-y}(1-x+y),
$$

which again are easy to check.

This means that systems (2) and (4) are completely integrable, because they are defined in $\mathbb{R}^{3}$ and they have two independent first integrals. We recall that any $n$-dimensional linear differential system having $n-1$ independent first integrals, is completely integrable, see for instance [5].

It is well known that, in general, the analytic study of the periodic orbits is not easy because they are non-local objects. Sometimes the crossing periodic orbits of the discontinuous piecewise differential systems are studied computing the fixed points of the Poincaré return map, see for instance $[4,8]$. But the computation of the Poincaré return map is in general difficult because we need to know the times that orbits, starting at $x=0$, need to return to 
$x=0$. Here using the first integrals of the completely integrable discontinuous piecewise differential systems we shall study their crossing periodic orbits, this tool already has been used previously in piecewise linear differential systems, see $[7,9]$, here we also use it for piecewise nonlinear differential systems.

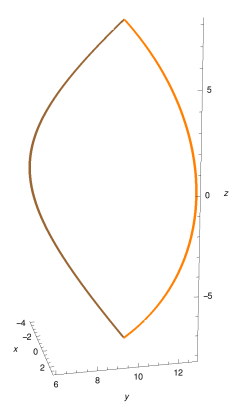

FiguRE 1. The crossing periodic orbit of Theorem 1 which intersects the plane $x=0$ in the points $(0,10,8)$ and $(0,10,-8)$.

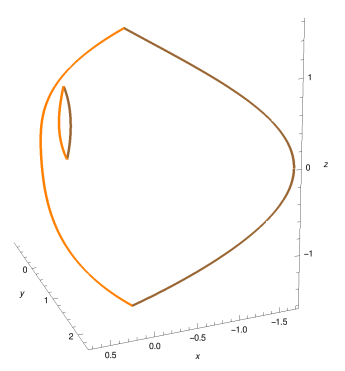

FiguRE 3. The crossing periodic orbits of Theorem 2 which intersects the plane $x=0$ the first one at the points $(0,-0.76803 \ldots, 0.5) \quad$ and $(0,-0.76803 . .,-0.5) \quad$ and the second one at the points $(0,1.67834 . ., 1.57384 .$.$) and$ $(0,1.67834 . .,-1.57384 .$.$) .$

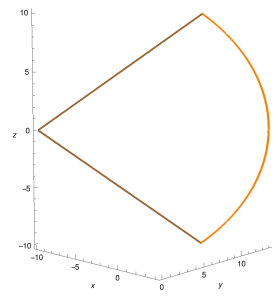

Figure 2. The homoclinic orbit of Corollary 1 which intersects the plane $x=0$ in the points $(0,10,10)$ and $(0,10,-10)$.

Our main results are the following.

Theorem 1. The discontinuous piecewise differential system (2) has a crossing periodic orbit which intersects the discontinuous plane $x=0$ in two points 
if and only if the points are $\left(0, y_{0}, z_{0}\right)$ and $\left(0, y_{0},-z_{0}\right)$ satisfying $z_{0} \neq 0, y_{0}>0$ and $y_{0}^{2}-z_{0}^{2}>0$. One of these periodic orbits is shown in Figure 1.

The proofs of Theorem 1 and its corollary are given in section 3 .

Corollary 1. For each pair of points $\left(0, y_{0}, \pm y_{0}\right)$ such that $y_{0}>0$, the system (2) has a homoclinic orbit to the equilibrium point $\left(-y_{0}, 0,0\right)$ that intersects the discontinuous plane $x=0$ first in $\left(0, y_{0}, y_{0}\right)$, and after in $\left(0, y_{0},-y_{0}\right)$. One of these homoclinic orbits is shown in Figure 2.

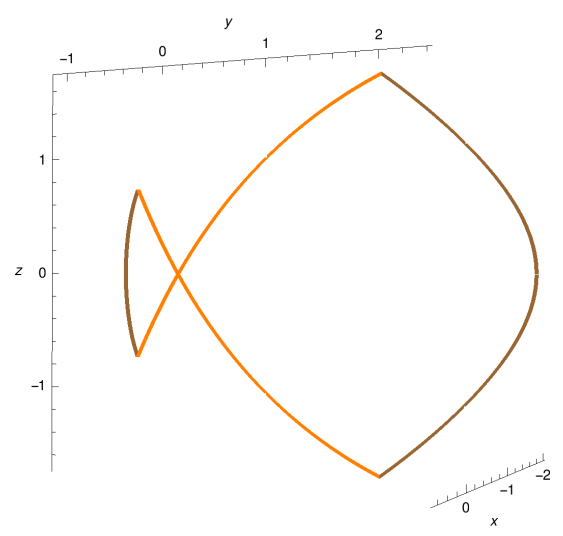

FiguRE 5. The homoclinic orbits of Corollary 2 which intersects the plane $x=0$ in the points $\left(0, y_{0},-y_{0}\right)$ and $\left(0, y_{1},-y_{1}\right)$, where $y_{0}=-0.76803$. and $y_{1}=1.67834 \ldots$

Theorem 2. The following statements hold.

(i) The discontinuous piecewise differential system (4) has a periodic orbit which intersects the discontinuous plane $x=0$ in two points if and only if the points are $\left(0, y_{0}, z_{0}\right)$ and $\left(0, y_{0},-z_{0}\right)$ satisfying $z_{0} \neq 0$, $y_{0}^{2}-z_{0}^{2}>0$ and $y_{0}$ is the solution of the equation $1+y-g e^{y}=0$ for $g<1$. We show two of these periodic orbits in Figure 3.

(ii) The discontinuous piecewise differential system (4) has a periodic orbit which intersects the discontinuous plane $x=0$ in four points if and only if the points are $\left(0, y_{0}, \pm z_{0}\right)$ and $\left(0, y_{1}, \pm z_{1}\right)$ satisfying $z_{i} \neq 0$, $y_{i}{ }^{2}-z_{i}{ }^{2}<0$ for $i=0,1, y_{0}$ is the negative solution of the equation $1+y-g e^{y}=0$ and $y_{1}$ is the positive solution of the equation $1+y-$ $g e^{y}=0$ for $0<g<1$. One of these periodic orbits is shown in Figure 4. 
The proofs of Theorem 2 and its corollary are given in section 3 .

Corollary 2. For each pair of points $\left(0, y_{0}, \pm y_{0}\right)$ such that $y_{0}$ is a solution of the equation $1+y-g e^{y}=0$ for $g<1$, system (4) has two homoclinic orbits to the equilibrium point $\left(1-e^{-y_{0}}\left(1+y_{0}\right), 0,0\right)$ that intersects the discontinuous plane $x=0$ first in $\left(0, y_{0}, y_{0}\right)$, and after in $\left(0, y_{0},-y_{0}\right)$. Two of these homoclinic orbits are shown in Figure 5.

\section{Proof of Theorem 1}

For studying the existence of crossing periodic orbits of the piecewise linear differential system (2) we must analyze the behavior of the two linear differential systems

$$
\dot{x}=z, \quad \dot{y}=z, \quad \dot{z}=y,
$$

for $x<0$, and

$$
\dot{x}=z, \quad \dot{y}=z, \quad \dot{z}=-y,
$$

for $x>0$.

From (3) two independent first integrals for system (6) are

$$
H_{1}(x, y, z)=y^{2}-z^{2} \text { and } F(x, y, z)=x-y,
$$

and two independent first integrals for system (7) are

$$
H_{2}(x, y, z)=y^{2}+z^{2} \text { and } F(x, y, z)=x-y .
$$

We want to study the intersection of the surfaces $H_{1}(x, y, z)=h_{1}$ with $F(x, y, z)=f$ in the half-space $x<0$. The curve defined by this intersection is denoted by $\gamma_{h_{1} f}$, and clearly this curve is formed by orbits of system (6) when the intersection is non-empty.

For $h_{1} \neq 0$ the surfaces $H_{1}(x, y, z)=h_{1}$ are hyperbolic cylinders, that is, their projections in the plane $x=0$ describe a hyperbola whose vertices are the points $\left(0, \pm \sqrt{h_{1}}, 0\right)$ if $h_{1}>0$, or the points $\left(0,0, \pm \sqrt{\left|h_{1}\right|}\right)$ if $h_{1}<0$. The surface $H_{1}(x, y, z)=0$ is formed by two planes whose intersection is the $x$-axis. Finally, the surfaces $F(x, y, z)=f$ are planes.

We observe that the curve $\gamma_{h_{1} f}$ is well-defined, i.e. the intersection $\left\{H_{1}=h_{1}\right\} \cap$ $\{F=f\} \cap\{x<0\}$ is always non-empty, and $\gamma_{h_{1} f}$ contains one orbit of system (6) contained in the half-space $x<0$ if $h_{1}>0$ and $f<\left|\sqrt{h_{1}}\right|$. Otherwise, when $h_{1}<0$ or when $h_{1}>0$ and $\left|\sqrt{h_{1}}\right| \leq f$, the curve $\gamma_{h_{1} f}$ contains two orbits of system (6) where each orbit is contained in distinct sheets of the hyperbolic cylinder if $h_{1}<0$ or if $h_{1}, f>0$ and $\sqrt{h_{1}} \leq f$ (see Figure 6), or both orbits 


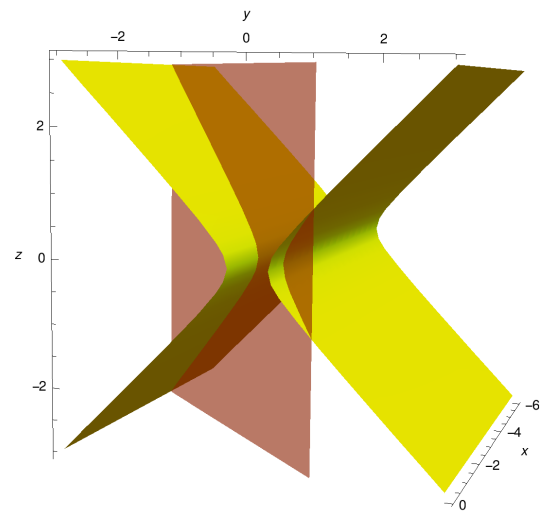

FiguRE 6. Intersection of the surfaces $H_{1}(x, y, z)=$ 0.1 and $F(x, y, z)=-1$ in the half-space $x<0$.

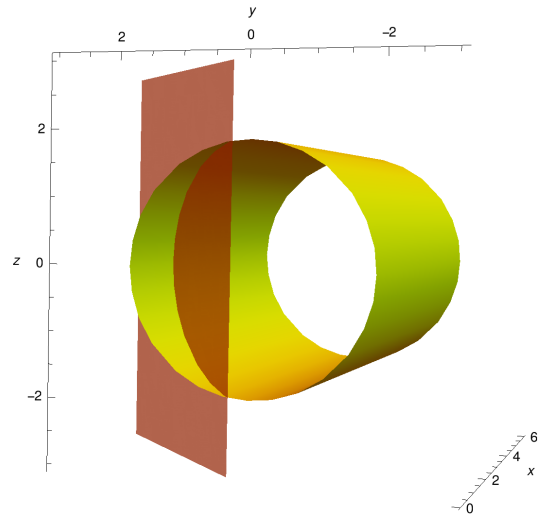

FiguRE 7. Intersection of the surfaces $H_{2}(x, y, z)=$ 3.5 and $F(x, y, z)=-0.3$ in the half-space $x>0$.

are contained in the same sheet of the hyperbolic cylinder if $h_{1}>0, f<0$ and $f \leq-\sqrt{h_{1}}$. Now, since $\gamma_{0}$ intersects the $x$-axis at the point $(f, 0,0)$, which is an equilibrium point of system (6), $\gamma_{0} f$ is formed by two, three or five orbits of system (6) when $f>0, f=0$ and $f<0$, respectively.

The surface $H_{2}=h_{2}>0$, is a cylinder whose projection in the plane $x=0$ is a circle with center at the origin and with radius $\sqrt{h_{2}}$. We want to study the intersection of $H_{2}(x, y, z)=h_{2}$ with $F(x, y, z)=f$ in the half-space $x>0$, denoted by $\gamma_{h_{2} f}$. First, we note that $\gamma_{h_{2} f}$ is empty if $-f<-\sqrt{h_{2}}$. If $-f \geq-\sqrt{h_{2}}$, the curve $\gamma_{h_{2} f}$ is formed by an orbit of system (7) if $h_{2}>0$, or by the equilibrium point $(f, 0,0)$ of system $(7)$ if $h_{2}=0$ (see the Figure 7 ).

A crossing periodic orbit of system (2) with two points in $x=0$ occurs only if an orbit of system (6) and an orbit of system (7) meet in the same two crossing points of $x=0$. So a periodic orbit of system (2) obtained from the connection of an orbit of $\gamma_{h_{1} f}$ with an orbit of $\gamma_{h_{2} f}$ is only possible if

$$
h_{1}>0 \text { and } f<0 \text {. }
$$

Let $\left(0, y_{0}, z_{0}\right)$ be a point such that $h_{1}=H_{1}\left(0, y_{0}, z_{0}\right), h_{2}=H_{2}\left(0, y_{0}, z_{0}\right)$ and $f=F\left(0, y_{0}, z_{0}\right)$ satisfying conditions (8). Considering the orbits of the systems

$$
H_{1}=h_{1}=y_{0}^{2}-z_{0}^{2}, \quad F=f=-y_{0}, \quad x=0,
$$

and

$$
H_{2}=h_{2}=y_{0}^{2}+z_{0}^{2}, \quad F=f=-y_{0}, \quad x=0,
$$


we obtain the points of the curves $\gamma_{h_{1} f}$ and $\gamma_{h_{2} f}$ that are in the plane $x=0$. The orbits satisfying both systems intersect the plane $x=0$ at the points $\left(0, y_{0}, \pm z_{0}\right)$ such that $y_{0}^{2}-z_{0}^{2}>0$ and $y_{0}>0$. Finally, $z_{0} \neq 0$ because the crossing periodic orbit must intersect the plane $x=0$ in two distinct points. So the "if" part of Theorem 1 is proved.

Now we shall prove the sufficient conditions of Theorem 1. Consider two points $\left(0, y_{0}, \pm z_{0}\right)$ which satisfy $y_{0}{ }^{2}-z_{0}^{2}>0, y_{0}>0$ and $z_{0} \neq 0$. Our objective is to prove that the orbits of systems (6) and (7) that intersect these points form a crossing periodic orbit of system (2). Taking the variable $x$ as a parameter the orbit of system (6) that intersects the plane $x=0$ at the points $\left(0, y_{0}, \pm z_{0}\right)$ is the curve $\left\{H_{1}=h_{1}\right\} \cap\{F=f\} \cap\{x \leq 0\}$, i.e.

$\left\{\left(x, x+y_{0}, \pm \sqrt{\left(x+y_{0}\right)^{2}-\left(y_{0}^{2}-z_{0}^{2}\right)}\right) ;-y_{0}+\sqrt{y_{0}^{2}-z_{0}^{2}} \leq x \leq 0\right\}$ in $x \leq 0$.

In a similar way the orbit $\left\{H_{2}=h_{2}\right\} \cap\{F=f\} \cap\{x \geq 0\}$ of system (7) which intersect the plane $x=0$ in those two points is

$$
\left\{\left(x, x+y_{0}, \pm \sqrt{\left(y_{0}^{2}+z_{0}^{2}\right)-\left(x+y_{0}\right)^{2}}\right) ; 0 \leq x \leq-y_{0}+\sqrt{y_{0}^{2}+z_{0}^{2}}\right\} \text { in } x \geq 0 .
$$

The connection of the curve (11) in $x \leq 0$ with the curve (12) in $x \geq 0$ provides a crossing periodic orbit. This completes the proof of Theorem 1.

Proof of Corollary 1. Solving systems (9) end (10) with respect to the variables $y_{0}$ and $z_{0}$ under the conditions $H_{1}\left(0, y_{0}, z_{0}\right)=0, H_{2}\left(0, y_{0}, z_{0}\right)=h_{2}$ and $F\left(0, y_{0}, z_{0}\right)=f$ with $f<0$, we obtain the points $\left(0, y_{0}, \pm y_{0}\right)$ with $y_{0}>0$.

The solution of system (6) with initial condition $\left(0, y_{0}, y_{0}\right)$ with $y_{0}>0$ is

$$
(x(t), y(t), z(t))=\left(\left(-1+e^{t}\right) y_{0}, e^{t} y_{0}, e^{t} y_{0}\right),
$$

for $t \in(-\infty, 0]$. We note that this orbit begins at the point $\left(-y_{0}, 0,0\right)$ which is equilibrium point of system (2), because all points in the $x$-axis are equilibrium points of that system.

The solution of system (7) with initial condition $\left(0, y_{0}, y_{0}\right)$ with $y_{0}>0$ is

$$
(x(t), y(t), z(t))=y_{0}(\cos t+\sin t-1, \cos t+\sin t, \cos t-\sin t),
$$

for $t \in[0, \pi / 2]$ and we have that $(x(\pi / 2), y(\pi / 2), z(\pi / 2))=\left(0, y_{0},-y_{0}\right)$.

Finally, the solution of system (6) with initial condition $\left(0, y_{0},-y_{0}\right)$ with $y_{0}>0$ is

$$
(x(t), y(t), z(t))=\left(\left(-1+e^{-t}\right) y_{0}, e^{-t} y_{0},-e^{-t} y_{0}\right),
$$

for $t \in[0,+\infty)$, and it is ends at the equilibrium point $\left(-y_{0}, 0,0\right)$ when $t=\infty$. 
In summary, for each $y_{0}>0$ system (2) has a homoclinic orbit to the equilibrium point $\left(-y_{0}, 0,0\right)$ obtained from the connection of the three pieces of orbits described above.

\section{Proof of Theorem 2}

Following the approach used to prove Theorem 1 we go to study the discontinuous piecewise differential system formed by the two differential systems

$$
\dot{x}=(x-y) z, \quad \dot{y}=z, \quad \dot{z}=-y,
$$

for $x>0$, and

$$
\dot{x}=(x-y) z, \quad \dot{y}=z, \quad \dot{z}=y,
$$

for $x<0$, by analyzing the intersections of the level surfaces of the two independent first integrals of each system. From (5) the two independent first integrals of system (13) are

$$
H_{1}(x, y, z)=y^{2}-z^{2} \text { and } G(x, y, z)=e^{-y}(1-x+y),
$$

and the two independent first integrals of system (14) are

$$
H_{2}(x, y, z)=y^{2}+z^{2} \text { and } G(x, y, z)=e^{-y}(1-x+y) .
$$

Since $G$ is a first integral for both systems, first we go to study the behavior of the surfaces $G=g$ for all $g \in \mathbb{R}$. As $G$ does not depended on $z$, for studying these surfaces it is sufficient to analyze the curve $G=\left.g\right|_{z=0}$, denoted by $\gamma(g)$. We have that the curve $\gamma(g)$ is defined by the equation $f_{g}(y)=x$ with $f_{g}(y)=1+y-g e^{y}$. This curve coincides with the graphic $\left(f_{g}(y), y\right)$ of the function $f_{g}: \mathbb{R} \rightarrow \mathbb{R}$.

For $g=0$ the curve $\gamma(g)$ is a straight line which pass through the points $(1,0)$ and $(0,-1)$, and the surface $G=0$ is the plane $1-x+y=0$.

When $g>0$, we define $y_{0}=\log (1 / g)$, and $y_{0}$ is a maximum of the function $f_{g}$, i.e. $f_{g}\left(y_{0}\right)=\log (1 / g) \geq f_{g}(y)$ for all $y \in \mathbb{R}$. Moreover, we observe that for $g=1, f_{g}\left(y_{0}\right)=y_{0}=0$. So the graphic of $f_{g}$ intersects the $y$-axis exactly at the point $(0,0)$, that is $\gamma(g)$ is contained in the half-space $x \leq 0$. Additionally if $g>1$ then $f_{g}\left(y_{0}\right)=y_{0}<0$. In this case the graphic of the function $f_{g}$ does not intersect the $y$-axis. Although for $0<g<1, f_{g}\left(y_{0}\right)=y_{0}>0$, i.e. the graphic of the function $f_{g}$ intersects the $y$-axis at two points, denoted by $\alpha(g)$ and $\beta(g)$, where $-1<\alpha(g)<0$ and $\beta(g)>0$.

We observed that when $y \rightarrow-\infty$ the graphic of the function $f_{g}$ tends to the straight line $x-y-1=0$ for any $g \in \mathbb{R}$, and when $y \rightarrow+\infty$ we have that $f_{g}(y) \rightarrow+\infty$ if $g \leq 0$, else if $g>0$ then $f_{g}(y) \rightarrow-\infty$. So if $g \leq 0$ and 
since for all $y \in \mathbb{R}$ we have that $f_{g}^{\prime}(y)>0$, the graphic of the $f_{g}$ intersects the $y$-axis in a unique point, denoted by $\rho(g) \leq-1$. We note that $\rho(0)=-1$.

In summary we have that the system

$$
e^{-y}(1-x+y)=g, \quad x=0,
$$

has in $\mathbb{R}^{2}$ only the solution $(0, \rho(g))$ if $g \leq 0$; two solutions $(0, \alpha(g))$ and $(0, \beta(g))$ if $0<g<1$; the trivial solution $(0,0)$ if $g=1$; and it has no solutions if $g>1$.

Varying $z$ we shall describe the behavior of the surface $G=g$ for all $g \in \mathbb{R}$. First we have that the solutions of system (15) considered in $\mathbb{R}^{3}$ are the straight line $y=\rho(g)$ if $g \leq 0$, the straight lines $y=\alpha(g)$ and $y=\beta(g)$ if $0<g<1$, and the straight line $y=0$ if $g=1$.

Now we must analyze the intersection of the surfaces $H_{1}(x, y, z)=h_{1}$ and $G(x, y, z)=g$ in the half-space $x>0$, denoted by $\gamma_{h_{1} g}$. Using the above analysis and taking the projections of these surfaces onto the plane $x=0$, it is easy to check that $\gamma_{h_{1} g}$ is empty when either $g \geq 1$, or $h_{1}>0$, or $-\sqrt{h_{1}}<\alpha(g)$ and $\beta(g)<\sqrt{h_{1}}$.

When $\gamma_{h_{1} g}$ is not empty it is formed by one or two orbits of system (13) if $h_{1} \neq 0$. More precisely, $\gamma_{h_{1} g}$ is one orbit of the system contained in the half-space $x>0$, i.e. which does not intersect the plane $x=0$ if $h_{1}>0$ and $\rho(g)=-\sqrt{h_{1}}$; and $\gamma_{h_{1} g}$ is also one orbit of the system when $h_{1}>0$, $\alpha(g)=-\sqrt{h_{1}}$ and $\beta(g)>\sqrt{h_{1}}$, but in this case it intersects the plane $x=0$ at the points $\left(0, \beta(g), \pm \sqrt{\beta(g)^{2}-h_{1}}\right)$. Otherwise $\gamma_{h_{1} g}$ contains two orbits of system (13). However, when

(a) $h_{1}>0$ and $\rho(g)<-\sqrt{h_{1}}$ one of the orbits of the system is contained in the half-space $x>0$ and the other orbit intersects the plane $x=0$ at the points $\left(0, \rho(g), \pm \sqrt{\rho(g)^{2}-h_{1}}\right)$;

(b) $h_{1}>0, \alpha(g)<-\sqrt{h_{1}}$ and $\beta(g)>\sqrt{h_{1}}$ both orbits intersect the plane $x=0$ in two points; one orbit at the points $\left(0, \alpha(g), \pm \sqrt{\alpha(g)^{2}-h_{1}}\right)$, and the other orbit at the points $\left(0, \beta(g), \pm \sqrt{\beta(g)^{2}-h_{1}}\right)$ (see Figure 8); 


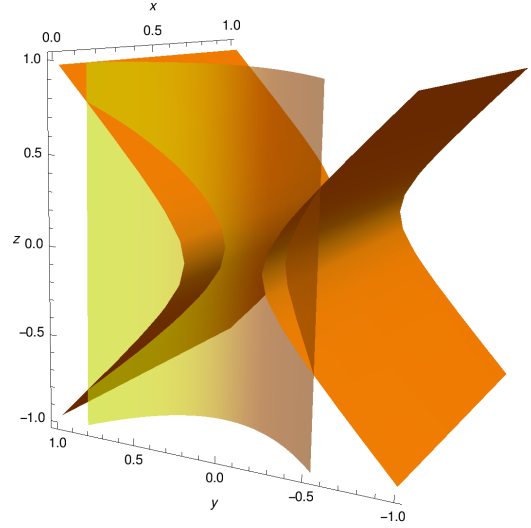

FiguRE 8. Intersection of the surfaces $H_{1}=0.05$ and $G=0.8$ in the half-space $x>0$.

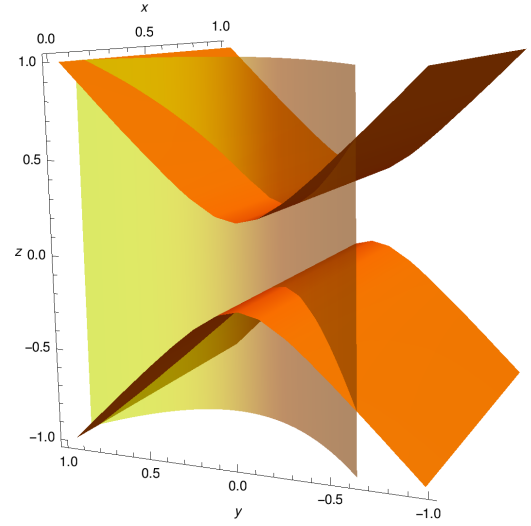

Figure 9. Intersection of the surfaces $H_{1}=-0.05$ and $G=0.7$ in the half-space $x>0$.

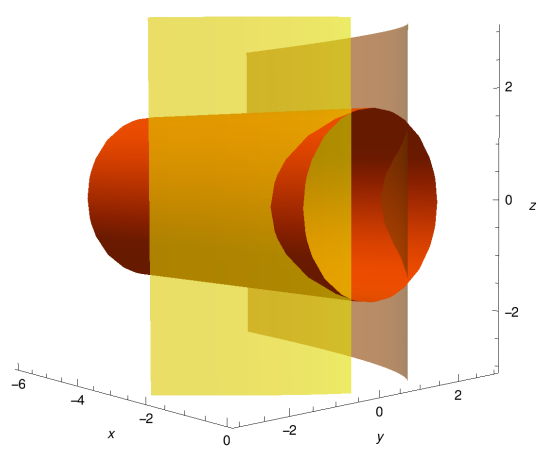

FiguRE 10. Intersection of the surfaces $H_{2}=2.5$ and $G=0.8$ in the half-space $x<0$.

(c) $h_{1}<0$ and $g \leq 0$ both orbits intersect the plane in only one point: an orbit does it at the point $\left(0, \rho(g), \sqrt{\rho(g)^{2}-h_{1}}\right)$, and the other orbit does it at the point $\left(0, \rho(g),-\sqrt{\rho(g)^{2}-h_{1}}\right)$

(d) $h_{1}<0$ and $0<g<1$ one of the orbits is contained in the positive sheet of the hyperbolic cylinder and it intersects the plane $x=0$ at the points 
$\left(0, \alpha(g), \sqrt{\alpha(g)^{2}-h_{1}}\right)$ and $\left(0, \beta(g), \sqrt{\beta(g)^{2}-h_{1}}\right)$. The other orbit is contained in the negative sheet of the hyperbolic cylinder and this orbit intersects the plane $x=0$ at the points $\left(0, \alpha(g),-\sqrt{\alpha(g)^{2}-h_{1}}\right)$ and $\left(0, \beta(g),-\sqrt{\beta(g)^{2}-h_{1}}\right)$, as shown in Figure 9.

When $h_{1}=0$ the curve $\gamma_{h_{1} g}$ is formed by five orbits of system (13) for any $g$ because the surface $G=g$ intersects the $x$-axis at the point $(1-g, 0,0)$ which is an equilibrium point of system (4). Moreover, when $g \leq 0$ two of these orbits intersect the plane $x=0$ : one orbit at the point $(0, \rho(g), \rho(g))$ and the other at the point $(0, \rho(g),-\rho(g))$; when $0<g<1$ each orbit intersects the plane $x=0$ at one point, namely: $(0, \alpha(g),-\alpha(g)),(0, \alpha(g), \alpha(g))$, $(0, \beta(g),-\beta(g))$ and $(0, \beta(g),-\beta(g))$.

On the other hand using again the analysis of the function $f_{g}$ and looking at the projections of the surfaces $H_{2}(x, y, z)=h_{2}$ and $G(x, y, z)=g$ onto the plane $x=0$ we have that $\gamma_{h_{2} g}=\left\{H_{2}=h_{2}\right\} \cap\{G=g\} \cap\{x<0\}$ is not empty when $|\rho(g)| \leq \sqrt{h_{2}}$ or $|\alpha(g)| \leq \sqrt{h_{2}}$. So under these assumptions $\gamma_{h_{2} g}$ is formed by one orbit of system (14) if $g \leq 0$ and it intersects the discontinuous plane at the points $\left(0, \rho(g), \pm \sqrt{h_{2}-\rho(g)^{2}}\right)$, or the curve $\gamma_{h_{2} g}$ is formed by two orbits of system (14) if $0<g<1$ and each orbit has two points in the plane $x=0$ : one orbit at the points $\left(0, \alpha(g), \pm \sqrt{h_{2}-\alpha(g)^{2}}\right)$ and the other orbit at the point $\left(0, \beta(g), \pm \sqrt{h_{2}-\beta(g)^{2}}\right)$. For $g>1$, the curve $\gamma_{h_{2} g}$ is a closed orbit contained in a half-space $x<0$ (see Figure 10).

In order that system (4) has a crossing periodic orbit it is necessary that the orbits of system (13) connect with the orbits of system (14), i.e. the orbits for both systems must intersect the discontinuous plane $x=0$ at the same points. This is only possible if either

$$
h_{1}>0, \quad h_{2}>0 \text { and } g<1,
$$

or

$$
h_{1}<0, \quad h_{2}>0, \quad \text { and } \quad 0<g<1 .
$$

This means that taking a point $\left(0, y_{0}, z_{0}\right)$ in the plane $x=0$ such that $H_{1}\left(0, y_{0}, z_{0}\right)=h_{1}, H_{2}\left(0, y_{0}, z_{0}\right)=h_{2}$ and $G\left(0, y_{0}, z_{0}\right)=g$ satisfying conditions (16) and considering the solutions of the systems

$$
H_{1}=h_{1}=y_{0}^{2}-z_{0}^{2}, \quad G=g=e^{-y_{0}}\left(1+y_{0}\right), \quad x=0,
$$


and

$$
H_{2}=h_{2}=y_{0}^{2}+z_{0}^{2}, \quad G=g=e^{-y_{0}}\left(1+y_{0}\right), \quad x=0,
$$

we obtain the connection points of the curves $\gamma_{h_{1} g}$ and $\gamma_{h_{2} g}$, that they will be two points $\left(0, y_{0}, \pm z_{0}\right)$ such that $y_{0}^{2}-z_{0}^{2}>0$ and where $y_{0}$ is a solution of the equation $1+y-g e^{y}=0$ if $g \leq 1$, or they will be four points $\left(0, y_{0}, \pm z_{0}\right)$ and $\left(0, y_{1}, \pm z_{1}\right)$ satisfying the same conditions. That is in this second case the crossing periodic orbits appear in pairs.

Let $\left(0, y_{0}, z_{0}\right)$ be a point of the plane $x=0$ such that $H_{1}\left(0, y_{0}, z_{0}\right)=h_{1}$, $H_{2}\left(0, y_{0}, z_{0}\right)=h_{2}$ and $G\left(0, y_{0}, z_{0}\right)=g$ satisfying conditions (17), then the solutions of systems (18) and (19) are of the form $\left(0, y_{0}, \pm z_{0}\right)$ and $\left(0, y_{1}, \pm z_{1}\right)$ with $y_{i}{ }^{2}-z_{i}{ }^{2}<0$ and being $y_{i}$ a solution of the equation $1+y-g e^{y}=0$, $i=0,1$.

Again $z_{0} \neq 0$ because the crossing periodic orbit intersects the discontinuous plane at least in two distinct points and this completes the proof of the necessary conditions of statements (i) and (ii) of Theorem 2.

Now we present the proof of the sufficient conditions of these statements. Consider the two points $\left(0, y_{0}, \pm z_{0}\right)$ such that $z_{0} \neq 0, y_{0}^{2}-z_{0}^{2}>0$ and where $y_{0}$ is a solution of the equation $1+y-g e^{y}=0$ for some $g<1$. We will show that the orbits of systems (13) and (14) that intersect these points form a crossing periodic orbit of system (4). Taking the variable $y$ as a parameter we have that the orbits of system (13) and (14) that intersect the plane $x=0$ at the points $\left(0, y_{0}, \pm z_{0}\right)$ are respectively, the curves

$$
\left\{\left(1+y-g e^{y}, y, \pm \sqrt{\left(y_{0}^{2}+z_{0}^{2}\right)-y^{2}}\right) ;-\sqrt{y_{0}^{2}+z_{0}^{2}} \leq y \leq y_{0}\right\}
$$

in $x \leq 0$ and

$$
\left\{\left(1+y-g e^{y}, y, \pm \sqrt{y^{2}-\left(y_{0}^{2}-z_{0}^{2}\right)}\right) ; y_{0} \leq y \leq-\sqrt{y_{0}^{2}-z_{0}^{2}}\right\}
$$

in $x \geq 0$, if $y_{0}$ is the negative solution of the equation $1+y-g e^{y}=0$, or are the curves

$$
\left\{\left(1+y-g e^{y}, y, \pm \sqrt{y^{2}-\left(y_{0}^{2}-z_{0}^{2}\right)}\right) ; \sqrt{y_{0}^{2}-z_{0}^{2}} \leq y \leq y_{0}\right\}
$$

in $x \geq 0$ and

$$
\left\{\left(1+y-g e^{y}, y, \pm \sqrt{\left(y_{0}^{2}+z_{0}^{2}\right)-y^{2}}\right) ; y_{0} \leq y \leq \sqrt{y_{0}^{2}+z_{0}^{2}}\right\}
$$

in $x \leq 0$, if $y_{0}$ is the positive solution of the equation $1+y-g e^{y}=0$.

The connection of the orbit (20) in $x \leq 0$ with the orbit (21) in $x \geq 0$ provides a crossing periodic orbit of system (4) which intersects the discontinuous plane in two points. The same occurs for the connection of the orbit (22) in 
$x \geq 0$ with the orbit (23) in $x \leq 0$ and this completes the proof of statement (i) of Theorem 2.

Finally consider the points $\left(0, y_{0}, \pm z_{0}\right)$ and $\left(0, y_{1}, \pm z_{1}\right)$ satisfying the assumptions of statement (ii) of Theorem 2. Now the orbits of system (14) that intersect the plane $x=0$ in these points are two disjoint curves of the form

$$
\left\{\left(1+y-g e^{y}, y, \sqrt{y^{2}-\left(y_{0}^{2}-z_{0}^{2}\right)}\right) ; y_{0} \leq y \leq y_{1}\right\}
$$

and

$$
\left\{\left(1+y-g e^{y}, y,-\sqrt{y^{2}-\left(y_{0}^{2}-z_{0}^{2}\right)}\right) ; y_{0} \leq y \leq y_{1}\right\}
$$

in $x \geq 0$. We observed that the orbit $(24)$ stars at the point $\left(0, y_{0}, z_{0}\right)$ and ends at point $\left(0, y_{1}, z_{1}\right)$, and the orbit $(25)$ stars at the point $\left(0, y_{0},-z_{0}\right)$ and ends at point $\left(0, y_{1},-z_{1}\right)$. Therefore, connecting the orbits $(20),(24),(23)$ and (25) we obtained a crossing periodic orbit for system (4), see figure 5. This completes the proof of Theorem 2 .

Proof of Corollary 2. First we observed that all points in the $x$-axis are equilibrium points of system (4). According with the description of possible intersections of the surfaces $H_{1}(x, y, z)=h_{1}$ and $G(x, y, z)=g$ made previously, the existence of homoclinic orbits for system (4) is only possible if $h_{1}=0$.

Let $\left(0, y_{0}, y_{0}\right)$ be a point such that $y_{0}$ is a negative solution of the $g=$ $e^{-y}(1+y)$ for some $g<1$. Again, taking the variable $y$ as a parameter, we have that the orbits of system (14) that intersect the plane $x=0$ at the points $\left(0, y_{0}, y_{0}\right)$ are respectively, the curves

$$
\left\{\left(1+y-\left(1+y_{0}\right) e^{\left(y-y_{0}\right)}, y, \pm y\right) ; y_{0} \leq y \leq 0\right\} .
$$

We observe that when $y=0$, the two curves meet at the point $\left(1-e^{-y_{0}}\left(1+y_{0}\right)\right.$, $0,0)$ which is an equilibrium point of system (4). The same occurs when $y_{0}$ is the positive solution of the equation $g=e^{-y}(1+y)$ for some $g<1$, but the curves which describes the orbits of system (14) in this case are

$$
\left\{\left(1+y-\left(1+y_{0}\right) e^{\left(y-y_{0}\right)}, y, \pm y\right) ; 0 \leq y \leq y_{0}\right\} .
$$

Connecting the orbit (20) in $x \leq 0$ with the orbits (26) in $x \geq 0$ and connecting the orbit (22) in $x \leq 0$ with the orbits (27) in $x \geq 0$ we have two homoclinic orbits to the equilibrium point $\left(1-e^{-y_{0}}\left(1+y_{0}\right), 0,0\right)$.

\section{ACKNOWLEDGEMENTS}

The first author is partially supported by the Ministerio de Ciencia, Innovación y Universidades, Agencia Estatal de Investigación grants MTM201677278-P (FEDER), the Agència de Gestió d'Ajuts Universitaris i de Recerca 
grant 2017SGR1617, and the H2020 European Research Council grant MSCARISE-2017-777911. The second author is partially supported by CNPq-Brazil grant 443302/2014-6, PROCAD/CAPES grant 88881.0 68462/2014-01 and PRONEX/FAPEG grant 2017/10267000-508. The third author is partially supported by the Coordenação Aperfeiçoamento de Pessoal de Nível Superior - Brasil (CAPES) - Finance Code 001.

\section{REFERENCES}

[1] A. Andronov, A. Vitt and S. Khaikin, Theory of Oscillations, Pergamon Press, Oxford, 1966.

[2] E.A. BARBAShin, Introduction to the theory of stability. In: T.Lukes (ed) Translated from the Russian by Transcripta Service, London, Wolters-Noordhoff Publishing, Groningen, 1970.

[3] M.di Bernardo, C.J. Budd, A.R.P. Kowaleczyk, Piecewise-Smooth Dynamical Systems: Theory and Applications, Appl. Math. Sci. Series 163, Springer, London, 2008.

[4] V. Carmona, E. Freire and E. Ponce, Bifurcation of invariant cones in piecewise linear homogeneous systems, Internat. J. Bifur. Chaos Appl. Sci. Engrg. 15 (2005), 2469-2484.

[5] M. Falconi and J. Llibre, $n-1$ independent first integrals for linear differential systems in $\mathbb{R}^{n}$ and $\mathbb{C}^{n}$, Qual. Theory Dyn. Syst. 4 (2004), 233-254.

[6] A.F. Filippov, Differential equations with discontinuous right-and sides, translated from the Russian, Mathematics and its applications (Soviet Series), vol. 18, Kluwer Academic Publishers Group, Dordrecht, 1988.

[7] J. Llibre, E. NuÑez AND A.E. Teruel, Limit cycles for planar piecewise linear differential systems via first integrals, Qual. Theory Dyn. Syst. 3 (2002), 29-50.

[8] J. Llibre And E. PONCE, Three nested limit cycles in discontinuous piecewise linear differential systems with two zones, Dyn. Contin. Discrete Impuls Syst. Ser. B 19 (2012), 325-335.

[9] J. Llibre and M.A. Teixeira, Periodic orbits of continuous and discontinuous piecewise linear differential systems via first integrals, São Paulo J. Math. Sci. 12 (2018), $121-135$.

[10] D.J.W. Simpson, Bifurcations in Piecewise-Smooth Continuous Systems, World Scientific Series on Nonlinear Science A, vol 69, World Scientific, Singapore, 2010.

${ }^{1}$ Departament de Matemàtiques, Universitat Autònoma de Barcelona, 08193 Bellaterra, Barcelona, Catalonia, Spain.

Email address: jllibre@mat.uab.cat

${ }^{2}$ Institute of Mathematics and Statistics of Federal University of Goiás, Avenida Esperança s/n, Campus Samambaia, CeP 74690-900, Goiânia, Goiás, BRAZIL.

Email address: djtonon@ufg.br 
${ }^{3}$ Institute of Mathematics and Statistics of Federal University of Goiás, Avenida Esperança s/n, Campus Samambaia, CeP 74690-900, Goiânia, Goiás, BRAZIL.

Email address: marianaqueirozvelter@gmail.com 